\title{
Changes in Trend of Newly Prescribed Anti-Glaucoma Medications in Recent Nine Years in a Japanese Local Community
}

\author{
Kenji Kashiwagi*
}

Department of Community and Family Medicine and Department of Ophthalmology, Interdisciplinary School of Medicine and Engineering, University of Yamanashi, Yamanashi 409-3898, Japan

\begin{abstract}
Purpose: To investigate changes in the trend of anti-glaucoma ophthalmic solutions in 2000-2008 in a Japanese local community.

Materials and Methods: The subjects of this study were patients with glaucoma who were registered in the database of the glaucoma clinic of University of Yamanashi Hospital or the database of the Association for Research in Supporting System of Chronic Diseases (ARSSCD). The trend of newly prescribed anti-glaucoma ophthalmic solutions from January 2000 through July 2008 was analyzed. Changes in the trend of anti-glaucoma ophthalmic solutions and the persistence of each anti-glaucoma ophthalmic solution were investigated.

Results: A total of 1,955 glaucoma patients were enrolled. Mean intraocular pressure (IOP) significantly reduced by year $\left(\mathrm{R}^{2}=0.7938, \mathrm{p}<0.001\right)$. The number of new prescriptions for analysis in the study period was 7,831 . Prostaglandin (PG) analogues accounted for approximately $40 \%$ of the prescriptions during the period, and were followed by beta-blockers and carbonic anhydrase inhibitors (CAIs). Those three anti-glaucoma ophthalmic solutions accounted for approximately $90 \%$ of all the prescriptions. Latanoprost showed the longest persistence among the anti-glaucoma ophthalmic solutions. The number of newly prescribed anti-glaucoma ophthalmic solutions gradually increased with time.

Conclusions: PG analogues are the most frequently prescribed and latanoprost showed the longest persistence among the anti-glaucoma ophthalmic solutions. PG analogues, beta-blockers, and CAIs accounted for the majority of newly prescribed anti-glaucoma ophthalmic solutions. An increase in the number of newly prescribed anti-glaucoma ophthalmic solutions may contribute to further IOP reduction.
\end{abstract}

Keywords: Glaucoma, anti-glaucoma ophthalmic solutions, prostaglandin, beta-blocker, carbonic anhydrase inhibitor, Japanese.

\section{INTRODUCTION}

Intraocular pressure (IOP) reduction is the best therapeutic choice for glaucoma and sufficient IOP reduction is required to maintain visual function. There are two options for glaucoma treatment from the aspect to IOP reduction: medication and surgery. Surgery dramatically reduces IOP; however, it sometimes results in severe complications, such as cataract formation, hypotonic maculopathy, and blebrelated infection $[1,2]$. Therefore, the use of anti-glaucoma ophthalmic solutions is recommended in many glaucoma cases [3]. Recently, new anti-glaucoma ophthalmic solutions that can effectively reduce IOP were launched and other ophthalmic solutions are expected to be launched in the near future.

Glaucoma is a vision-threatening disease that requires lifelong care. In this regard, ophthalmologists need to devise a plan to treat patients in the long term. The treatment plan must be personalized for each patient. From this point of view, the Association for Research in Supporting System of

*Address correspondence to this author at the Department of Community and Family Medicine and Department of Ophthalmology, Interdisciplinary School of Medicine and Engineering, University of Yamanashi, 1110 Shimokato Chuo, Yamanashi 409-3898, Japan; Tel: 81-552-73-9657; Fax: 81-552-73-6757; E-mail: kenjik@yamanashi.ac.jp
Chronic Diseases (ARSSCD) was established in 2005. A new support system for patients with chronic diseases including glaucoma was set up in which limited data on proper care are shared among participating medical facilities through the Internet [4]. This system is considered to be an example of the electric health record systems. Important data for glaucoma management, such as IOP, medication, and visual field test, of registered patients measured at different registered medical facilities are collected into the database through the Internet. More than 1,400 patients are registered with this system so far. Two databases were employed in this study: one consists of the medical charts of University of Yamanashi Hospital and the other is this Web-based system.

It is important to know the trend of anti-glaucoma ophthalmic solutions and apply the trend to routine glaucoma treatment to keep in touch with current medication status. However, as there are few studies in Japan that investigated changes in the trend of anti-glaucoma ophthalmic solutions, this study attempted to investigate such changes using data from the above two systems.

\section{SUBJECTS AND METHODS}

This study was conducted in accordance with the Helsinki Declaration after receipt of approval from the Ethics Committee of Yamanashi University. Consent for the 
use of treatment data was obtained from the subjects prior to the study.

Patients registered with ARSSCD and glaucoma patients followed up by the glaucoma clinic of University of Yamanashi Hospital were enrolled in this study. Data of IOP reduction and newly prescribed anti-glaucoma ophthalmic solutions from January 2000 through July 2008 were investigated. Goldmann applanation tonometry was employed for IOP measurement. Ophthalmic solutions that were prescribed only once or for a period of less than one month were eliminated from the study. Investigated parameters were changes in the trend of anti-glaucoma ophthalmic solutions sorted by pharmacological action, changes in the trend of anti-glaucoma ophthalmic solutions having the same pharmacological action, persistence, and changes in the number of newly prescribed anti-glaucoma ophthalmic solutions. The above parameters were also investigated in patients with normal tension glaucoma (NTG). The IOPs of NTG patients never exceeded $21 \mathrm{mmHg}$ in any measurements, and the patients did not have a history of extensive blood loss or head trauma causing visual field loss and disc cupping. They were confirmed not to have any intra-cranial abnormalities that would contribute to isual field defect and optic nerve atrophy.

\section{Statistical Analysis}

Results are expressed as means \pm standard deviation. JMP (version 5, SAS Institute Inc., NC) was used for statistical analysis.

\section{RESULTS}

\section{Enrolled Subjects}

A total of 1,955 patients with glaucoma were enrolled in this study. Nine hundred and fifty were male and 1,005 were female, and the mean age was $66.0 \pm 16.5$ yrs. The number of newly prescribed ophthalmic solutions for analysis in the study period was 7,831. The distributions of glaucoma subtypes were similar during the study period. Primary open angle glaucoma accounted for approximately half of the total glaucoma types throughout the study period. NTG was the second most popular subtype, its frequency being approximately one-quarter of the total glaucoma types. Secondary glaucoma and other subtypes accounted for approximately another one-quarter of the total glaucoma types.

\section{Changes in Mean IOP}

During the study period, a total of 52,176 IOP measurements were performed. Mean IOP showed a significant reduction by year $(\mathrm{p}<0.0001$, Tukey-Kramer test). Mean IOP in 2000 was $16.7 \pm 4.1 \mathrm{mmHg}$, while that in 2008 was $15.1 \pm 3.7 \mathrm{mmHg}$. There was a significant correlation between mean IOP and year $\left(R^{2}=0.7938, p<0.001\right)$ (Fig. 1).

\section{Changes in Trend of Anti-Glaucoma Ophthalmic Solutions Sorted by Pharmacological Action}

Among the anti-glaucoma ophthalmic solutions, prostaglandin (PG) analogues were the most frequently prescribed, accounting for $40 \%$ to $45 \%$ during the study period. Beta-blockers were the second most frequently prescribed and carbonate anhydrase inhibitors (CAIs) followed in the early part of the study period. During followup, the prescription frequency of beta-blockers decreased and that of CAIs increased. PG analogues, beta-blockers, and CAIs accounted for approximately $90 \%$ of all anti-glaucoma ophthalmic solutions prescribed during the period (Fig. 2).

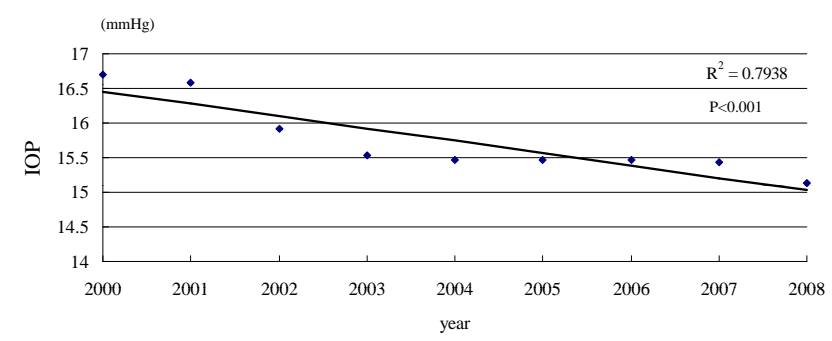

Fig. (1). Changes in intraocular pressure by year. Pearson's correlation coefficient was calculated. IOP: intraocular pressure.

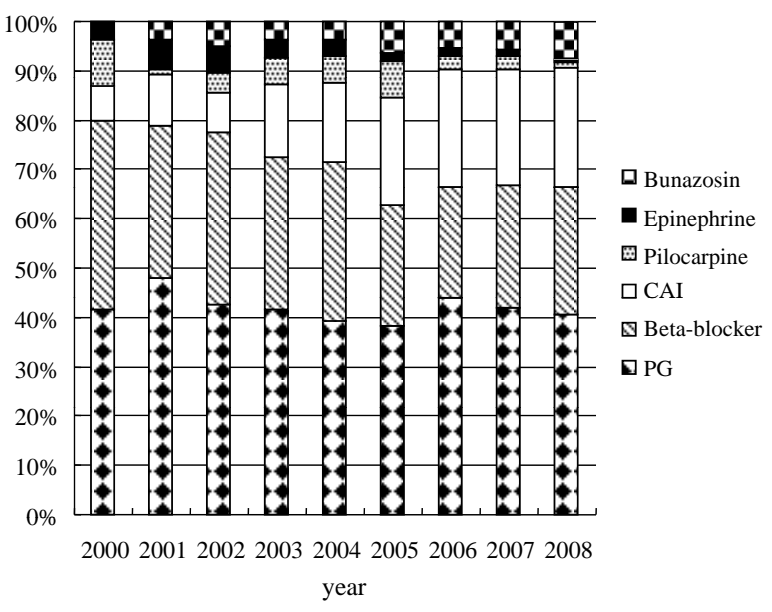

Fig. (2). Changes in trend of anti-glaucoma ophthalmic solutions sorted by pharmacological action. CAI: carbonic anhydrase inhibitor, PG: prostaglandin.

\section{Changes in Trend of Anti-Glaucoma Ophthalmic Solutions having the Same Pharmacological Action}

Among the PG analogues, latanoprost accounted for approximately $90 \%$ from 2000 to 2006 , but its prescription frequency was reduced to $72.5 \%$ in 2008 one year after the launch of the new PG analogue, travaprost (Fig. 3a).

Among the beta-blockers, timolol accounted for approximately $60 \%$ to $70 \%$ during the study period, followed by carteolol. The prescription frequency of other beta-blockers did not show significant changes during the period (Fig. 3b).

\section{Persistence of Anti-Glaucoma Ophthalmic Solutions}

Ophthalmic solutions prescribed in 2001 were investigated for persistence. PG analogues showed the longest persistence among all types of anti-glaucoma ophthalmic solutions. PG analogues were prescribed for a significantly longer period of $5.3 \pm 2.4$ yrs than beta-blockers (5.0 $\pm 2.7 \mathrm{yrs})$ and CAIs $(3.8 \pm 3.1 \mathrm{yrs})$. Latanoprost showed the longest persistence of $5.8 \pm 2.2$ yrs among all types of anti-glaucoma ophthalmic solutions (Fig. 4). 


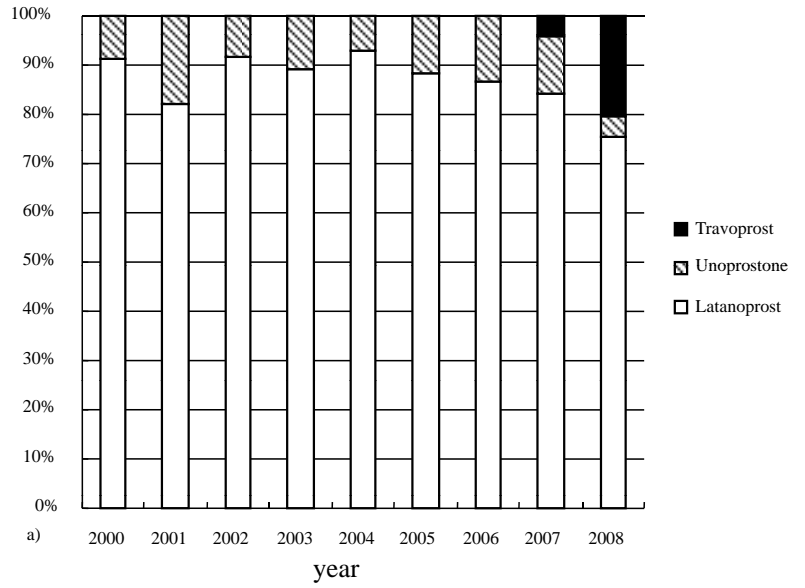

Fig. (3). Changes in trend of anti-glaucoma ophthalmic solutions having the same pharmacological action.

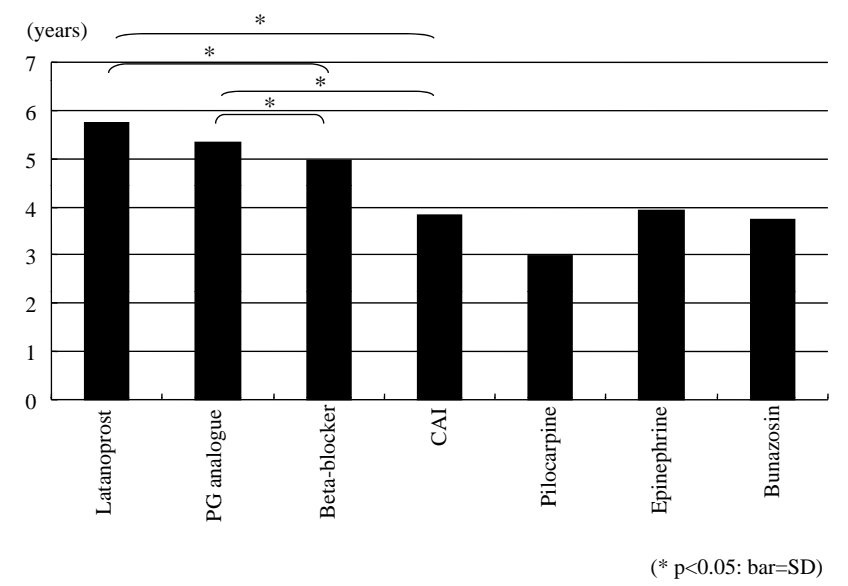

Fig. (4). Persistence of anti-glaucoma ophthalmic solutions. $\mathrm{p}<$ 0.05 , one-way repeated measures ANOVA and post hoc analysis with the Tukey-Kramer test. CAI: carbonic anhydrase inhibitor, PG: prostaglandin, SD: standard deviation.

\section{Changes in the Number of Newly Prescribed Anti- Glaucoma Ophthalmic Solutions}

The number of newly prescribed anti-glaucoma ophthalmic solutions per glaucoma patient ranged from 1.5 to 1.8 during the study period and the frequency slightly increased with time (Fig. 5).

\section{Changes in Trend and Persistence of Anti-Glaucoma Ophthalmic Solutions among NTG Patients}

The number of NTG patients was 249 (113 males and 136 females) and the number of newly prescribed ophthalmic solutions was 453. Fig. (6) shows the averaged frequency of anti-glaucoma ophthalmic solutions during the study period. PG analogues accounted for approximately $50 \%$, followed by beta-blockers and CAIs. Those three antiglaucoma ophthalmic solutions accounted for $95 \%$ of all new prescriptions. Then, ophthalmic solutions prescribed from 2001 to 2004 were investigated for their persistence (Fig. 7). Latanoprost showed a significantly longer prescription period of $4.6 \pm 1.0 \mathrm{yrs}$ than beta-blockers $(3.9 \pm 1.7 \mathrm{yrs})$ and CAIs (3.4 \pm 1.4 yrs).

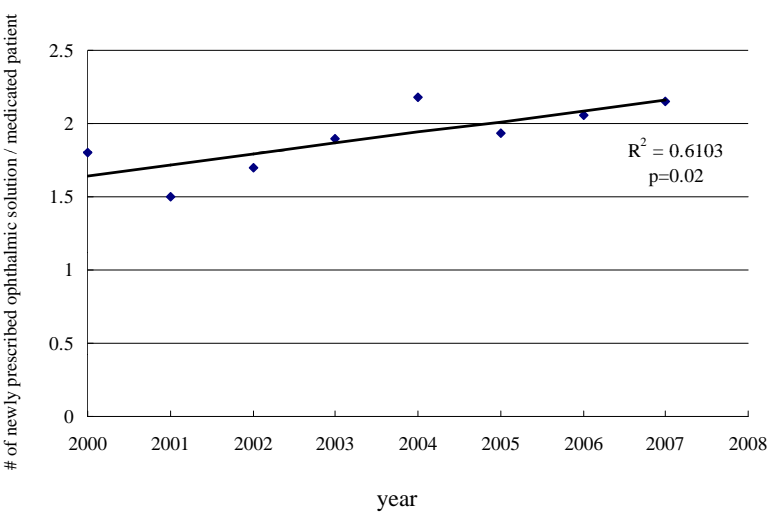

Fig. (5). Changes in the number of newly prescribed anti-glaucoma ophthalmic solutions. Pearson's correlation coefficient was calculated.

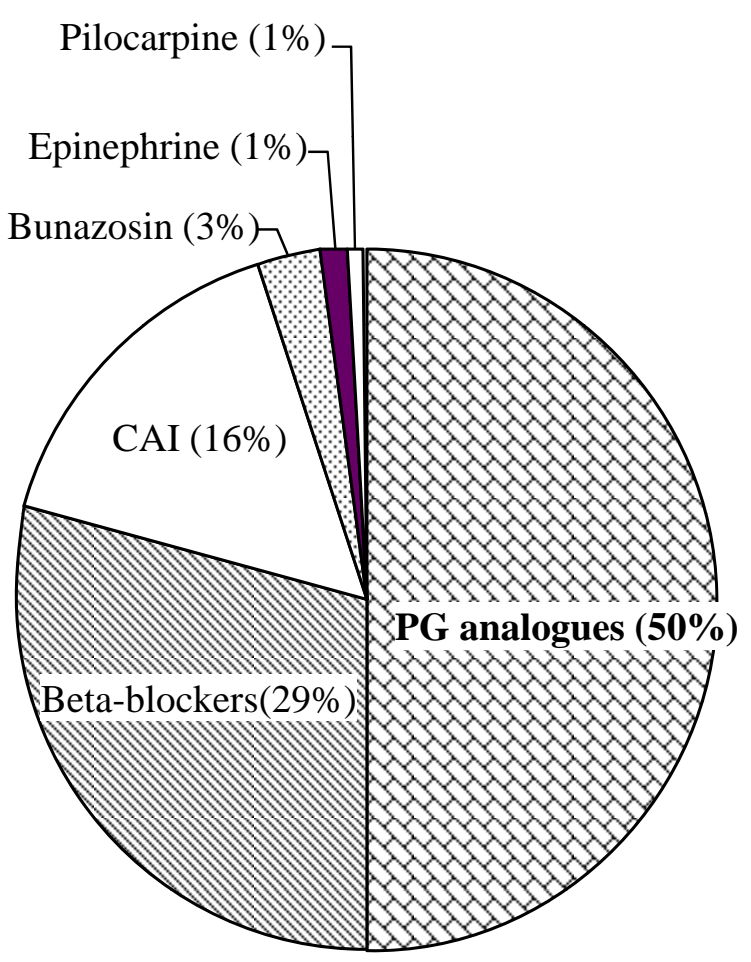

Fig. (6). Average prescription frequency of anti-glaucoma ophthalmic solutions in NTG patients. NTG: normal tension glaucoma, PG: prostaglandin, CAI: carbonic anhydrase inhibitor

\section{DISCUSSION}

IOP reduction is the most effective therapy for glaucoma. Several new anti-glaucoma ophthalmic solutions were launched in recent years. The Japan Glaucoma Society recommends topical treatment as the first-line treatment for glaucoma [3]. Changes in the trend of IOP control and antiglaucoma ophthalmic solutions from 2000 through 2008 were investigated. It is important to investigate the trend of IOP control and anti-glaucoma ophthalmic solutions with a large population because glaucoma often requires lifelong treatment. 


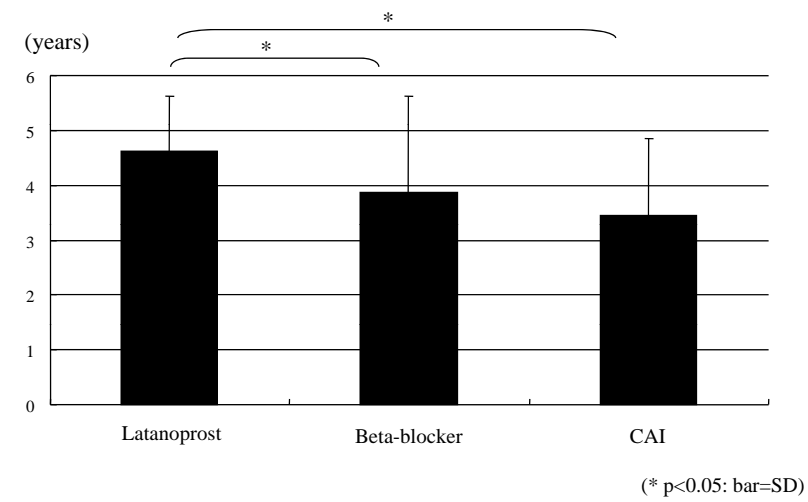

Fig. (7). Persistence of anti-glaucoma ophthalmic solutions among NTG patients. ${ }^{*} \mathrm{p}<0.05, \mathrm{p}<0.05$, one-way repeated measures ANOVA and post hoc analysis with the Tukey-Kramer test. NTG: normal tension glaucoma, CAI: carbonic anhydrase inhibitor.

The increased reduction of mean IOP by year is an indication that ophthalmologists now recognize the fact that much greater IOP reduction than ever is required to maintain visual function in glaucoma patients. This result also indicates that improvements in medical therapy enable further IOP reduction.

Although the Japan Glaucoma Society recommended beta-blockers and PG analogues as the first-line treatment for glaucoma [3], this study revealed that PG analogues, in particular latanoprost, were the most frequently used antiglaucoma ophthalmic solutions. Anti-glaucoma ophthalmic solutions are required to possess the following characteristics: excellent potential for IOP reduction, good tolerance, and little adverse effects. PG analogues, in particular latanoprost, are considered to be a good candidate [5-7].

Beta-blockers occupied second place during the early part of the study period, but CAIs moved up to second place in the later part of the study period. CAIs significantly reduce IOP in 24 hours, while beta-blockers have low potential to reduce IOP at night [8-12]. It is recommended that IOP be kept at a sufficiently low level for 24 hours to prevent progression of glaucomatous optic neuropathy [3]. The trend of anti-glaucoma ophthalmic solutions was similar between all types of glaucoma and NTG. PG analogues, beta-blockers, and CAIs accounted for $90 \%$ of all the prescribed anti-glaucoma ophthalmic solutions.

Among the beta-blockers, timolol was the most frequently prescribed during the study period. It has been reported that there is no significant difference in IOP reduction among the beta-blockers [13-15]. Timolol has the longest history as an anti-glaucoma ophthalmic solution and sufficient data of its adverse effects are available. Several modifications of timolol, including the once-a-day type and the generic type, were launched in recent years. These may explain why timolol is the most frequently prescribed among the beta-blockers.

In Japan, only two PG analogues were available before 2007: isopropyl unoprostone and latanoprost. Latanoprost shows superior IOP reduction compared with isopropyl unoprostone [16-18]. However, the launch of travoprost reduced the frequency of latanoprost prescription. In the near future, other PG analogues, such as bimatoprost and tafluprost, will be released in Japan. Although it is not clear which PG analogue shows superior IOP reduction or the least adverse effects [19-21]. PG analogues on the whole are expected to be the most frequently prescribed anti-glaucoma ophthalmic solutions. However, the frequency of latanoprost prescription is expected to be reduced.

As anti-glaucoma ophthalmic solutions are usually used for a very long time or even a lifetime, it is desirable that they have high persistence and adherence [22]. It has been reported that latanoprost has high persistence and adherence compared to other ophthalmic solutions [23]. The current study revealed that latanoprost has the highest persistence among all types of anti-glaucoma ophthalmic solutions. The persistence of latanoprost is excellent for all types of glaucoma. Latanoprost, unoprostone, and beta-blockers were the first-line anti-glaucoma ophthalmic solutions in 2001. Therefore, latanoprost may have the highest persistence among the first-line anti-glaucoma ophthalmic solutions. CAIs and other drugs are used as second-line or third-line drugs except in very limited cases and the first-line drug usually shows the highest persistence regardless of adherence. The number of potential first-line anti-glaucoma ophthalmic solutions is increasing. Therefore, the real persistence of ophthalmic solutions should be determined using another method.

The current study revealed that the number of antiglaucoma ophthalmic solutions gradually increased during the follow-up period. Modern glaucoma therapy requires much greater IOP reduction that ever. It has been reported that $30 \%$ IOP reduction from the baseline was preferred in patients with NTG [24]. However, it is not easy to reduce IOP by $30 \%$ with only one anti-glaucoma ophthalmic solution, particularly in the case of NTG. This could be one reason why the number of anti-glaucoma ophthalmic solutions gradually increased during the follow-up period. Another reason is that any combinations of anti-glaucoma solutions are not authorized by the Japanese government.

The subjects of the study are mainly residents of Yamanashi Prefecture and neighboring areas. Therefore, the current results may not represent the national trend of antiglaucoma ophthalmic solutions. It is necessary to conduct a similar study using a much larger number of patients from a wider area. Furthermore, it is necessary to investigate the effects of changes in the trend of anti-glaucoma ophthalmic solutions on the extent of IOP reduction or visual function.

The current study demonstrated that the development of new anti-glaucoma ophthalmic solutions has contributed to the reduction of IOP to a much greater extent than ever. New anti-glaucoma ophthalmic solutions scheduled for release in the near future are expected to contribute to the maintenance of visual function in glaucoma patients. However, the emergence of new treatment may lead to some negative issues, such as the increased possibility of adverse effects and the economic burden to not only the patient but also the society. Clearly, there is a need to pursue the appropriate medication for glaucoma patients while paying attention to the above-described issues. 


\section{ACKNOWLEDGEMENTS}

The author appreciates the support extended by the Association for Research in Supporting System of Chronic Diseases. This study was supported in part by grants from the Ministry of Education, Culture, Sports, Science and Technology, Japan, The Watanabe Memorial Foundation for the Advancement of Technology, and Pfizer Inc. Ltd.

\section{REFERENCES}

[1] Bindlish R, Condon GP, Schlosser JD, D'Antonio J, Lauer KB, Lehrer R. Efficacy and safety of mitomycin-C in primary trabeculectomy: five-year follow-up. Ophthalmology 2002; 109: 1336-4.

[2] Jongsareejit B, Tomidokoro A, Mimura T, Tomita G, Shirato S, Araie M. Efficacy and complications after trabeculectomy with mitomycin C in normal-tension glaucoma. Jpn J Ophthalmol 2005; 49: 223-7.

[3] The Japan Glaucoma Society Guidelines for Glaucoma. $2^{\text {nd }}$ ed. Nippon Ganka Gakkai Zasshi 2006; 110: 778-814.

[4] Kashiwagi K. Developing the new Supporting System of Chronic Diseases using the internet. Nippon Ganka Gakkai Zasshi 2007; 111: 114-6.

[5] Vetrugno M, Cantatore F, Ruggeri G, et al. Primary open angle glaucoma: an overview on medical therapy. Prog Brain Res 2008; 173: 181-93.

[6] Arranz-Marquez E, Teus MA. Prostanoids for the management of glaucoma. Expert Opin Drug Saf 2008; 7: 801-8.

[7] Denis P, Lafuma A, Khoshnood B, Mimaud V, Berdeaux G. A meta-analysis of topical prostaglandin analogues intra-ocular pressure lowering in glaucoma therapy. Curr Med Res Opin 2007; 23: 601-8.

[8] Costagliola C, Parmeggiani F, Virgili G, et al. Circadian changes of intraocular pressure and ocular perfusion pressure after timolol or latanoprost in Caucasians with normal-tension glaucoma. Graefes Arch Clin Exp Ophthalmol 2007; 15: 15.

[9] Nakamoto K, Yasuda N. Effect of concomitant use of latanoprost and brinzolamide on 24-hour variation of IOP in normal-tension glaucoma. J Glaucoma 2007; 16: 352-7.

[10] Stewart WC, Konstas AG, Nelson LA, Kruft B. Meta-analysis of 24-hour intraocular pressure studies evaluating the efficacy of glaucoma medicines. Ophthalmology 2008; 115: 1117-22.
[11] Orzalesi N, Rossetti L, Invernizzi T, Bottoli A, Autelitano A. Effect of timolol, latanoprost, and dorzolamide on circadian IOP in glaucoma or ocular hypertension. Invest Ophthalmol Vis Sci 2000; 41: 2566-73.

[12] Liu JH, Kripke DF, Weinreb RN. Comparison of the nocturnal effects of once-daily timolol and latanoprost on intraocular pressure. Am J Ophthalmol 2004; 138: 389-95.

[13] Gandolfi SA, Cimino L. Beta-adrenergic antagonists in the treatment of glaucoma. Eur J Ophthalmol 2001; 11: S63-6.

[14] Drance SM. Introductory comments on potential differences between beta-blockers in the treatment of open-angle glaucoma. Surv Ophthalmol 1999; 43: S173-5.

[15] Zimmerman TJ. Topical ophthalmic beta blockers: a comparative review. J Ocul Pharmacol 1993; 9: 373-84.

[16] Enoki M, Saito J, Hara M, Uchida T, Sagara T, Nishida T. Additional reduction in intraocular pressure achieved with latanoprost in normal-tension glaucoma patients previously treated with unoprostone. Jpn J Ophthalmol 2006; 50: 334-7.

[17] Tsukamoto H, Mishima HK, Kitazawa Y, Araie M, Abe H, Negi A. A comparative clinical study of latanoprost and isopropyl unoprostone in Japanese patients with primary open-angle glaucoma and ocular hypertension. J Glaucoma 2002; 11: 497-501.

[18] Otori $\mathrm{Y}$, Tokugawa $\mathrm{H}$, Morimura $\mathrm{H}$, et al. The effect of substituting latanoprost $0.005 \%$ for unoprostone $0.12 \%$. Nippon Ganka Gakkai Zasshi 2004; 108: 207-12.

[19] Yildirim N, Sahin A, Gultekin S. The effect of latanoprost, bimatoprost, and travoprost on circadian variation of intraocular pressure in patients with open-angle glaucoma. J Glaucoma 2008; 17: 36-9.

[20] Kumar RS, Istiantoro VW, Hoh ST, Ho CL, Oen FT, Aung T. Efficacy and safety of a systematic switch from latanoprost to travoprost in patients with glaucoma. J Glaucoma 2007; 16: 606-9.

[21] Denis P, Launois R, Devaux M, Berdeaux G. Comparison of diurnal intraocular pressure control by latanoprost versus travoprost : results of an observational survey. Clin Drug Investig 2006; 26: 703-14.

[22] Morley AM, Murdoch I. The future of glaucoma clinics. Br J Ophthalmol 2006; 90: 640-5.

[23] Nordstrom BL, Friedman DS, Mozaffari E, Quigley HA, Walker AM. Persistence and adherence with topical glaucoma therapy. Am J Ophthalmol 2005; 140: 598-606.

[24] C. n.-t. g. s. group. The effectiveness of intraocular pressure reduction in the treatment of normal-tension glaucoma. Collaborative Normal-Tension Glaucoma Study Group. Am J Ophthalmol 1998; 126: 498-505.

This is an open access article licensed under the terms of the Creative Commons Attribution Non-Commercial License (http: //creativecommons.org/licenses/by$\mathrm{nc} / 3.0 /$ ) which permits unrestricted, non-commercial use, distribution and reproduction in any medium, provided the work is properly cited. 